\title{
The Relationship between TP53 Gene Status and Carboxylesterase 2 Expression in Human Colorectal Cancer
}

\author{
Momoko Ishimine, ${ }^{1}$ Hyeon-Cheol Lee $\mathbb{D}^{1},{ }^{1}$ Hirofumi Nakaoka, ${ }^{2}$ Hajime Orita $\mathbb{D}^{3,4}$ \\ Toshiyuki Kobayashi, ${ }^{5}$ Konomi Mizuguchi, ${ }^{3,4}$ Mikumi Endo, ${ }^{4}$ Ituro Inoue, ${ }^{2}$ Koichi Sato, ${ }^{3,4}$ \\ and Takehiko Yokomizo ${ }^{1}$
}

\author{
${ }^{1}$ Department of Biochemistry, Juntendo University School of Medicine, Tokyo, Japan \\ ${ }^{2}$ Division of Human Genetics, National Institute of Genetics, Shizuoka, Japan \\ ${ }^{3}$ Department of Surgery, Juntendo Shizuoka Hospital, Juntendo University School of Medicine, Shizuoka, Japan \\ ${ }^{4}$ Shizuoka Medical Research Center for Disaster, Juntendo University, Shizuoka, Japan \\ ${ }^{5}$ Department of Pathology and Oncology, Juntendo University School of Medicine, Tokyo, Japan
}

Correspondence should be addressed to Hyeon-Cheol Lee; h-lee@juntendo.ac.jp

Received 16 October 2017; Accepted 28 December 2017; Published 31 January 2018

Academic Editor: Andreas Hillenbrand

Copyright (c) 2018 Momoko Ishimine et al. This is an open access article distributed under the Creative Commons Attribution License, which permits unrestricted use, distribution, and reproduction in any medium, provided the original work is properly cited.

Irinotecan (CPT-11) is an anticancer prodrug that is activated by the carboxylesterase CES2 and has been approved for the treatment of many types of solid tumors, including colorectal cancer. Recent studies with cell lines show that CES2 expression is regulated by the tumor suppressor protein p53. However, clinical evidence for this regulatory mechanism in cancer is lacking. In this study, we examined the relationship between TP53 gene status and CES2 expression in human colorectal cancer. Most colorectal cancer specimens (70\%; 26 of 37) showed lower CES2 mRNA levels ( $\geq 1.5$-fold lower) than the adjacent normal tissue, and only 30\% (12 of 37) showed similar ( $<1.5$-fold lower) or higher CES2 mRNA levels. However, TP53 gene sequencing revealed no relationship between CES2 downregulation and TP53 mutational status. Moreover, while colorectal cancer cells expressing wild-type p53 exhibited p53-dependent upregulation of CES2, PRIMA-1 ${ }^{\mathrm{MET}}$, a drug that restores the transcriptional activity of mutant p53, failed to upregulate CES2 expression in cells with TP53 missense mutations. These results, taken together, suggest that CES2 mRNA expression is decreased in human colorectal cancer independently of p53.

\section{Introduction}

Irinotecan (camptothecin-11 (CPT-11)) is a topoisomerase I inhibitor that has been used in the treatment of a wide spectrum of cancer, particularly colorectal cancer. Irinotecan is a prodrug, and its conversion to the active compound 7-ethyl-10-hydroxycamptothesin (SN-38) requires its hydrolysis by the carboxylesterase CES2. This enzyme exhibits a 60 -fold higher hydrolytic efficiency against irinotecan than CES1, another human carboxylesterase isozyme [1], and the ability of colorectal tumors to hydrolyze irinotecan correlates with their expression of CES2, but not CES1 [2].

Although the liver is the major site for drug metabolism in general, it has been suggested that local (i.e., intratumoral) activation may also contribute to the efficacy of irinotecan in colorectal cancer [2-4]. Indeed, the colon may also serve as a major site for irinotecan activation since CES2 is highly expressed in this tissue, while the expression of the other carboxylesterase isozymes is relatively low [2]. A pharmacokinetic study revealed that the majority of intravenously injected irinotecan is excreted in the feces without being metabolized, indicating that a considerably high concentration of intact irinotecan is present in the colorectal lumen [5]. Thus, CES2 expression in colorectal cancer could be a key determinant of the therapeutic efficacy of this drug.

Recent studies suggest that CES2 expression is regulated by p53 in colorectal cancer cell lines $[6,7]$. p53 is a tumor suppressor that is activated by a number of cellular stresses such as DNA damage, oxidative stress, and hypoxia. Approximately $50 \%$ of colorectal cancer bears missense mutations in 
TP53, the gene encoding p53. Immunohistochemistry (IHC) has been used in clinical settings to detect p 53 mutations based on the premise that mutated p53 protein accumulates in the nucleus due to its impaired degradation [8]. Thus, if deregulated p53 signaling affects CES2 expression in colorectal cancer, accumulation of mutated p53 protein could be a potential diagnostic biomarker for predicting the therapeutic efficacy of irinotecan.

With these possibilities in mind, we investigated the relationship between TP53 gene status and CES2 expression in colorectal cancer. Although CES2 expression was significantly lower in colorectal cancer specimens than in adjacent normal tissue, no clear correlation was observed between TP53 gene status and CES2 expression. These results demonstrate the complexity of the regulatory mechanisms controlling CES2 expression in human colorectal cancer.

\section{Materials and Methods}

2.1. Cell Culture. The human colorectal cancer cell lines HCT116, HCT C, LoVo, RKO, and KM12C were provided by Dr. Tatsuro Irimura (Juntendo University). LS174T, Caco-2, and SW480 were from the American Type Culture Collection (ATCC). All cells were cultured at $37^{\circ} \mathrm{C}$ and $5 \% \mathrm{CO}_{2}$ in a $1: 1(\mathrm{v} / \mathrm{v})$ mixture of Dulbecco's modified Eagle's medium (Wako) and Ham's F-12 medium (Wako) supplemented with $10 \%$ fetal bovine serum and penicillin/ streptomycin. Cells expressing wild-type p53 (HCT116, HCT C, LoVo, RKO, and LS174T) $[9,10]$ and p53-null Caco-2 [11] cells were seeded at $2 \times 10^{5}$ cells/well in 6-well plates and incubated for 24 hours. The cells were then treated with $10 \mu \mathrm{M}$ nutlin-3a (AdooQ BioScience) for 24 hours. Cells expressing mutant p53 (KM12C and SW480) $[12,13]$ were seeded at $4 \times 10^{5}$ cells/well in 6-well plates and incubated for 24 hours. The cells were then treated with $120 \mu \mathrm{M}$ PRIMA-1 ${ }^{\mathrm{MET}}$ (Tocris Bioscience) for 24 hours. The cells were washed twice with PBS and harvested by scraping.

2.2. Tumor and Adjacent Normal Tissue Specimens. All tumors and adjacent normal tissues were obtained from patients undergoing surgery at the Department of Surgery, Juntendo Shizuoka Hospital, Juntendo University School of Medicine. The study was approved by the Medical Research Ethics Committee of Juntendo Shizuoka Hospital (ethics approval number 457), and written informed consent was obtained from all patients.

2.3. Real-Time Reverse Transcriptase PCR. Total RNA from cell lines and tissues was extracted using the RNeasy Mini Kit (Qiagen) according to the manufacturer's protocol. One microgram (cells) or $0.2 \mu \mathrm{g}$ (tissue) of total RNA was reverse transcribed with random hexamers using SuperScript III (ThermoFisher Scientific) to generate cDNA. Semiquantitative real-time PCR was performed using FastStart Essential DNA Green Master (Roche) on a LightCycler 96 (Roche). GAPDH (cell lines except for LoVo) and 18S rRNA (LoVo and tissues) were used as reference genes. PCR reactions were performed in duplicate for all genes. The relative expression of each gene was calculated using the $2^{-\Delta \Delta \mathrm{Ct}}$ method. The sequences of the primers used were as follows: CES2 forward $5^{\prime}$-GTAGCACATTTTCAGTGTTCC- $3^{\prime}$ and reverse $5^{\prime}$-GTAGTTGCCCCCAAAGAA-3', 221 forward $5^{\prime}$-GATT TCTACCACTCCAAACGCC- $3^{\prime}$ and reverse $5^{\prime}$-AGAAGA TGTAGAGCGGGC- ${ }^{\prime}$, Noxa forward $5^{\prime}$-GCTGGAAGTC GAGTGTGCTA- $3^{\prime}$ and reverse $5^{\prime}$-CCTGAGCAGAAGAG TTTGGA- $3^{\prime}$, GAPDH forward $5^{\prime}$-TGCCCTCAACGACC ACTTTG- $3^{\prime}$ and reverse $5^{\prime}$-CTCTTCCTCTTGTGCTCTT GCTG- $3^{\prime}$, and $18 S$ rRNA forward $5^{\prime}$-GTAACCCGTTG AACCCCATT- $3^{\prime}$ and reverse $5^{\prime}$-CCATCCAATCGGTA GTAGCG-3'.

2.4. Sequencing. DNA was extracted from each tumor sample using the AllPrep DNA/RNA Mini Kit (Qiagen) according to the manufacturer's protocol. Sanger sequencing was performed using the BigDye Terminator Cycle Sequencing V3.1 Ready Reaction kit (ThermoFisher Scientific) on an ABI 3130xl Genetic Analyzer (Applied Biosystems). The oligonucleotide primers used are shown in Table S1. TP53 genotypes of the colorectal cancer were categorized as nonfunctional, partially functional, or functional using the International Agency of Research on Cancer (IARC) TP53 mutation database (release R18) [14].

2.5. Plasmid Construction and Transfection. Human CES2 cDNA with a stop codon was amplified by PCR from NCI-H226 cell cDNA library with primers hCES2_cloning forward $5^{\prime}$-TAGTTAAGCTTATGACTGCTCAGTCCCGC TC- $3^{\prime}$ and hCES2_cloning_reverse $5^{\prime}$-GGCCCTCTAGA CTACAGCTCTGTGTGTCTCT- ${ }^{\prime}$ and was cloned into the pcDNA3.1/Myc-His vector using HindIII and XbaI sites. HEK293T cells were transfected with the plasmid DNA or empty vector control using polyethylenimine Max (MW 40,000) (Polysciences, Warrington, PA).

2.6. Gel-Based Activity-Based Protein Profiling Analysis. Cells and tissues were homogenized in PBS and centrifuged at $100,000 \times \mathrm{g}$ for $45 \mathrm{~min}$ at $4^{\circ} \mathrm{C}$. The soluble proteomes $(15 \mu \mathrm{g}$ in $30 \mu \mathrm{L}$ of PBS) were incubated with $1 \mu \mathrm{M}$ fluorophosphonate-rhodamine probe for $30 \mathrm{~min}$ at $37^{\circ} \mathrm{C}$. After $30 \mathrm{~min}$, reactions were quenched with $4 \mathrm{x}$ SDS/PAGE loading buffer (reducing), separated by SDS/PAGE $[10 \%$ $(w / v)$ acrylamide], and visualized using a Typhoon FLA 9500 (GE Healthcare Life Sciences). Images were analyzed with ImageQuant TL software (GE Healthcare Life Sciences). Rhodamine fluorescence is shown in gray scale.

\section{Results and Discussion}

3.1. Upregulation of CES2 in p53 Wild-Type Colorectal Cancer Cells. We first asked whether p53 activation enhances CES2 expression. Several colorectal cancer cell lines expressing wild-type p53 (HCT116, HCT C, LS174T, LoVo, and RKO) $[9,10]$ and a p53-null cell line (Caco-2) [11] were treated with nutlin-3a, which inhibits the interaction between p53 and the E3 ubiquitin ligase MDM2 and thereby directly activates p53 signaling without genotoxic side effects [15]. The expression of p21, a downstream target of p53, increased 


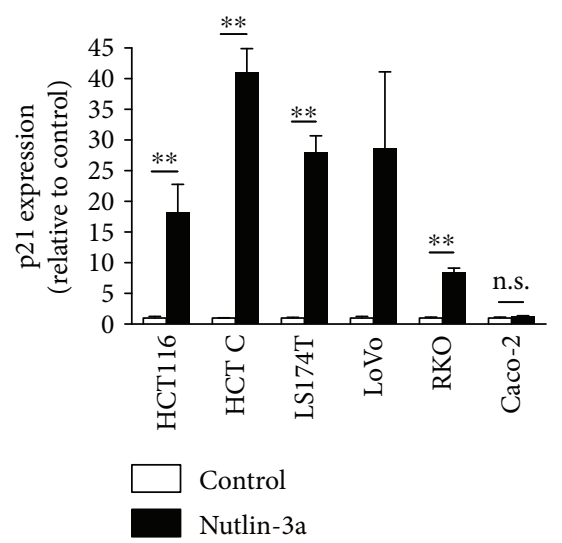

(a)

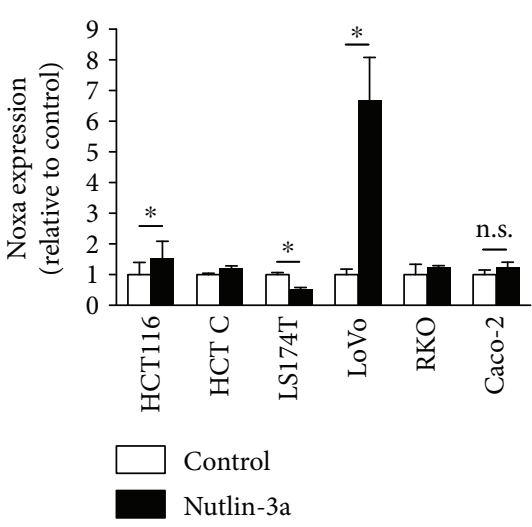

(b)

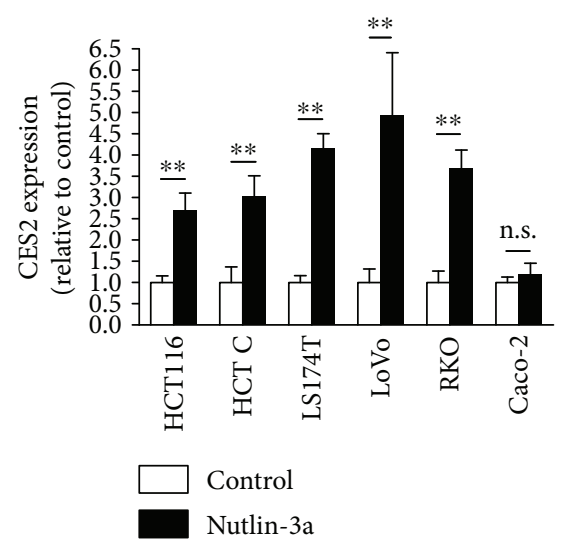

(c)

FIGURE 1: The expression of p21 (a), Noxa (b), and CES2 (c) was quantified by real-time reverse transcriptase PCR. Human colorectal cancer cell lines with wild-type p53 (HCT116, HCT C, LS174T, LoVo, and RKO) and p53-null Caco-2 cells were treated with $10 \mu \mathrm{M}$ nutlin-3a for 24 hours. For LoVo cells, $18 S$ rRNA was used as the reference gene. For other cell types, GAPDH was used as the reference gene. Data represent the mean values $\pm \operatorname{SEM}(n=3-4)$. ${ }^{*} P<0.05 ;{ }^{* *} P<0.01$; n.s.: no significance. A paired two-tailed $t$-test was used.

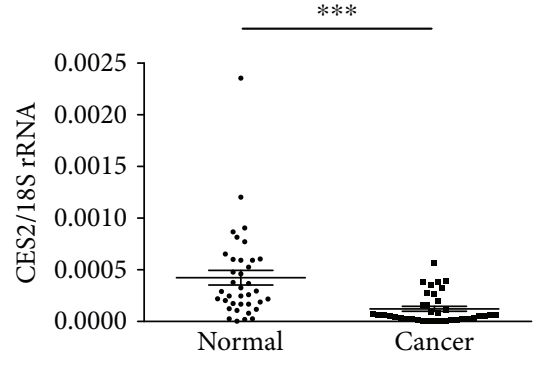

(a)

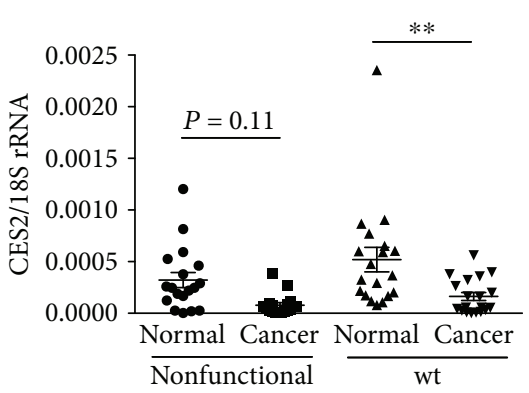

(b)

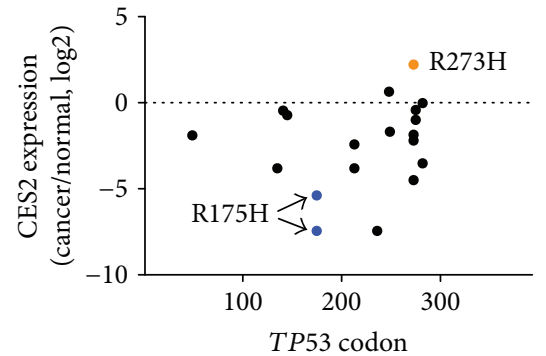

(c)

FIGURE 2: CES2 expression in human colorectal cancer and adjacent normal tissues was quantified by real-time reverse transcriptase PCR. (a) CES2 mRNA was significantly decreased in the tumor tissue. $18 \mathrm{~S} r R N A$ was used as a reference gene. ${ }^{* * *} P<0.001$ by paired two-tailed $t$-test. (b) The CES2 expression levels were compared between tumors with TP53 mutations generating nonfunctional p53 protein and tumors without TP53 mutations. ${ }^{* *} P<0.01$. A Tukey-Kramer test was used. (c) The relationship between CES2 expression and the position of TP53 mutations. The plot indicates the codon distribution of the TP53 missense mutations ( $x$-axis), and the samples' corresponding CES2 expression levels ( $y$-axis). The blue dots and the arrows indicate the samples with the $\mathrm{R} 175 \mathrm{H}$ mutation. The orange dot indicates the sample with the $\mathrm{R} 273 \mathrm{H}$ mutation.

following nutlin-3a treatment in all p53 wild-type cell lines tested, indicating enhanced p53 pathway activation in those cells (Figure 1(a)) [16]. Nutlin-3a also enhanced the expression of Noxa, another downstream target of p53, in HCT116 and LoVo cells (Figure 1(b)) [16]. Furthermore, nutlin-3a significantly increased the expression of CES2 in all of the cell lines expressing wild-type p53 (Figure 1(c)). However, the expression of these genes did not change in response to nutlin-3a in p53-null Caco-2 cells (Figures 1(a)-1(c)). These results provide further evidence that CES2 expression is positively regulated by the p53 pathway in colorectal cancer cells.

\subsection{CES2 Expression and Activity in Human Colorectal} Cancer. Approximately 50\% of colorectal cancer is reported to have missense mutations in the TP53 gene. Most of those mutations are found in the region encoding the DNAbinding domain. Such mutated p53 not only has reduced
DNA-binding capacity but also exerts a dominant negative effect on wild-type p53 [17], thereby severely compromising p53 function. The deregulation of the p53 pathway caused by these missense mutations affects the expression of numerous downstream target genes in cancer $[16,18,19]$.

To evaluate the relationship between CES2 expression and TP53 gene status in colorectal cancer, CES2 mRNA levels were compared in colorectal cancer specimens and adjacent normal tissue collected from 37 patients. Most of the tumor samples $(70 \% ; 26$ of 37) had lower CES2 expression than adjacent normal tissue ( $\geq 1.5$-fold decrease), and only $30 \%$ (12 of 37$)$ had similar (<1.5-fold decrease) or higher CES2 mRNA levels (Table S2, Figure 2(a)). This result is largely in agreement with that of a previous study that reported that colorectal cancer expressed lower levels of CES2 protein than adjacent normal tissue [3].

Next, the TP53 gene in each of the tumor samples was sequenced using an ABI 3130xl Genetic Analyzer. TP53 


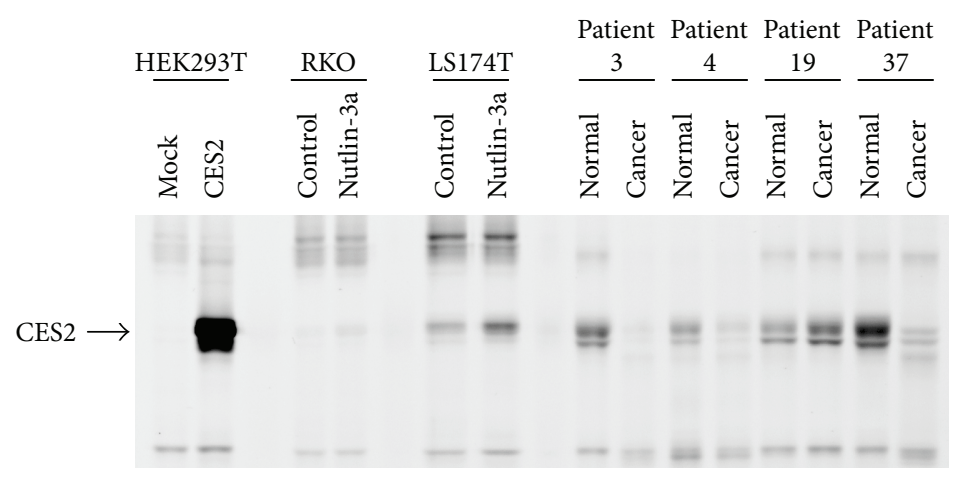

(a)

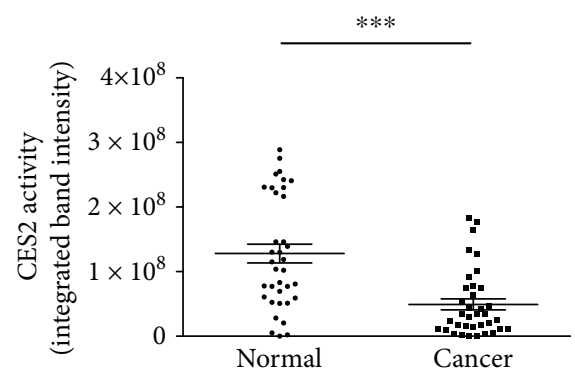

(b)

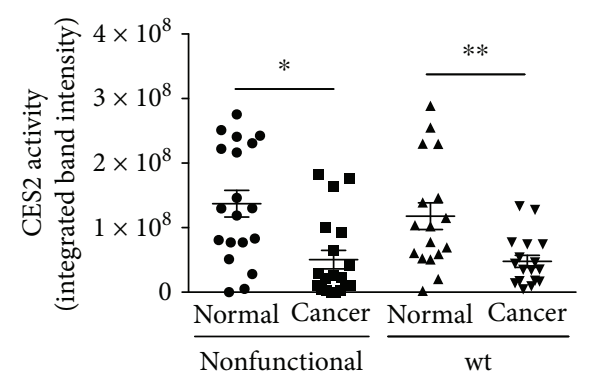

(c)

FIGURE 3: CES2 activity in human colorectal cancer and adjacent normal tissues was quantified by ABPP. (a) CES2 activities of HEK293T cells (mock versus CES2 overexpression), colorectal cancer cells (RKO and LS174T cells; control versus nutlin-3a), and representative human colorectal samples were shown. (b) CES2 activity was significantly decreased in the tumor tissue. ${ }^{* * *} P<0.001$ by paired two-tailed $t$-test. (c) The CES2 activities were compared between tumors with TP53 mutations generating nonfunctional p53 protein and tumors without TP53 mutations. ${ }^{*} P<0.05 ;{ }^{* *} P<0.01$. A Tukey-Kramer test was used.

mutations that reportedly result in a severe loss of p53 function were identified in $51 \%$ of the cancers (19 of 37) (Table S2), and all except one (18 of 19) were located in the region that encodes the DNA-binding domain of the p53 protein. Unexpectedly, the CES2 mRNA levels in the tumors with TP53 mutations resulting in nonfunctional p53 protein were comparable to those in the tumors without TP53 mutations $(P=0.85)$ (Figure 2(b)). These data suggest that the reduced expression of CES2 in colorectal cancer occurs independently of TP53 gene status. Indeed, while the two tumors with the R175H mutation (patients 5 and 6), one of the most common pathogenic loss-of-function TP53 mutations in colorectal cancer, showed a dramatic reduction in CES2 mRNA (42-fold and 174-fold, resp.) (Figure 2(c)), a sample with another common pathogenic mutation, R273H (patient number 14), exhibited higher CES2 expression (5-fold) in the tumor than in the adjacent normal tissue (Figure 2(c)). This also suggests the existence of alternative mechanisms enhancing CES2 expression even when the p53 pathway is inactivated.

We also measured the CES2 activity of the colorectal samples by activity-based protein profiling (ABPP) using a serine hydrolase activity probe fluorophosphonaterhodamine [20]. HEK293T cells expressing CES2 and colorectal cancer cells treated with nutlin-3a exhibited increased CES2 activity (Figure 3(a)). Similar to its expression, CES2 activity was lower in the tumors than in the adjacent normal tissue (Figures 3(a) and 3(b)), and the reduction of CES2 activity also occurred independently of TP53 gene status $(P=0.9995)$ (Figure 3(c)).

We finally examined the relationship between CES2 expression and p21 expression. We found that p21 expression was significantly reduced in the tumors compared with adjacent normal tissue (Figure S1 (a)) and that the reduction was also independent of the presence of TP53 mutation $(P=0.9997)$ (Figure S1 (b)). Notably, there was a significant positive correlation between $\mathrm{p} 21$ and CES2 expression even in the tumors with nonfunctional p53 (Table S3). It has been shown that $\mathrm{p} 21$ expression can be induced by various stress signals in a p53-independent fashion [21]. These data, taken together, suggest that CES2 expression is regulated in a p53independent manner that also controls p 21 expression in colorectal cancer.

3.3. CES2 Expression in Colorectal Cancer Cells with TP53 Missense Mutations. Recent studies identified small molecules that reactivate mutant p53 [22-25]. Although there was no clear relationship between CES2 expression and the presence of TP53 missense mutations in the colorectal cancer specimens in this study, restoration of p53 function might reactivate the p53 pathway, resulting in an upregulation of CES2 expression. To test this hypothesis, colorectal cancer cells harboring mutant $\mathrm{p} 53$ were treated with PRIMA-1 ${ }^{\mathrm{MET}}$, a p53-reactivating drug. This agent has been tested in a phase 


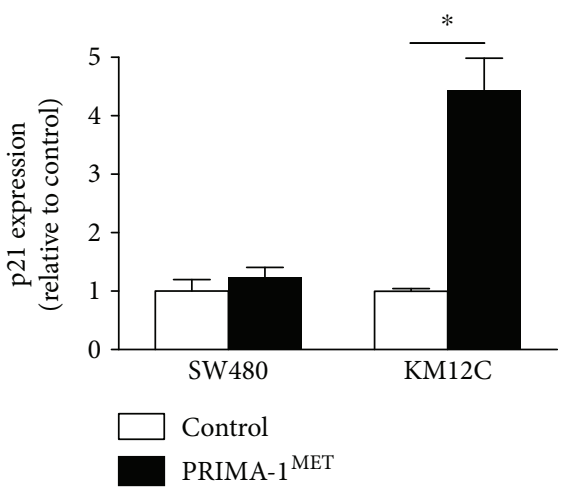

(a)

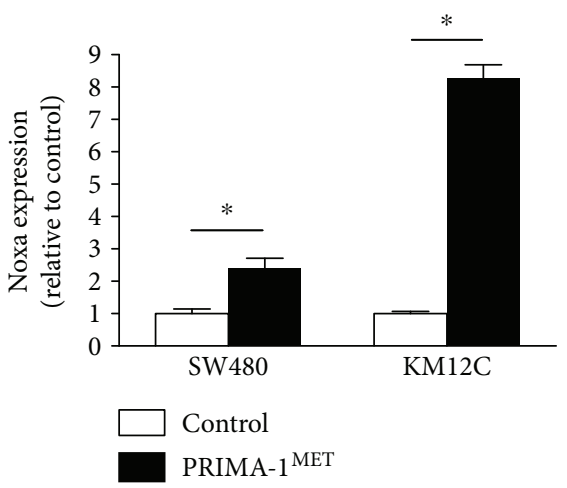

(b)

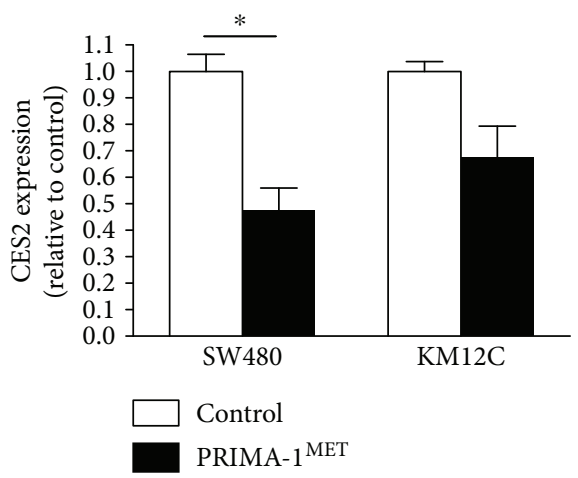

(c)

FIgURE 4: The expression of p21 (a), Noxa (b), and CES2 (c) was quantified by real-time reverse transcriptase PCR. Human colorectal cancer cell lines with TP53 missense mutations (SW480 and KM12C) were treated with $120 \mu \mathrm{M}$ PRIMA-1 ${ }^{\mathrm{MET}}$ for 24 hours. GAPDH was used as a reference gene. Data represent the mean values \pm SEM $(n=3-4) .{ }^{*} P<0.05$; n.s.: no significance. A paired two-tailed $t$-test was used.

I/IIa clinical trial for the treatment of hematologic malignancies and prostate cancer [26] and is currently being tested in a phase Ib/II clinical trial in patients with high-grade serous ovarian carcinoma. Among the cell lines tested, p21 expression was upregulated following PRIMA-1 ${ }^{\mathrm{MET}}$ treatment in KM12C (p53 H179R) [12] cells (Figure 4(a)), and Noxa expression was upregulated in response to PRIMA-1 ${ }^{\mathrm{MET}}$ in both SW480 (p53 R273H/P309S) [13] and KM12C cells (Figure 4(b)), suggesting that the reactivation of the p53 pathway occurred in these cells. However, CES2 expression was decreased in these cells after p53 pathway reactivation (Figure 4(c)). These data, taken together, provide further evidence that the regulation of CES2 expression is not straightforward and that mechanism(s) other than p53 are likely to be involved.

Shang et al. previously showed that CES2 expression is regulated by the xenobiotic-sensing transcription factor Pregnane X receptor (PXR) in HepG2 human hepatoma cells [27]. However, PXR agonism did not lead to upregulation of CES2 expression in colorectal cancer cells (LoVo, SW480, and KM12C) (data not shown), suggesting distinct mechanism(s) of CES2 regulation in colorectal cancer cells. Recent studies show that CES2 activates not only irinotecan but also other anticancer drugs such as gemcitabine [28], capecitabine [29], and pentyl PABC-Doxaz (PPD) [30]. Thus, understanding how CES2 expression is regulated in normal and cancerous cells will expand the utility of these anticancer drugs.

\section{Conclusions}

Most of the colorectal cancer specimens analyzed in this study showed a considerable reduction in CES2 expression compared with adjacent normal tissue. However, there was no obvious correlation between TP53 gene status and the reduction in CES2 expression. Thus, given the role of local CES2 expression in the activation of irinotecan, direct measurement of CES2 expression and/or activity (rather than TP53 mutational status or p53 protein expression) may be an effective method to predict the efficacy of irinotecan in colorectal cancer. Our results, however, do not rule out a potential role for wild-type p53, which could upregulate CES2 when activated by anticancer drugs. Further studies should be performed to fully understand the regulatory mechanisms of CES2 expression in cancer and the contribution of $\mathrm{p} 53$.

\section{Conflicts of Interest}

The authors declare no competing financial interest. 


\section{Authors' Contributions}

Momoko Ishimine and Hyeon-Cheol Lee contributed equally to this work.

\section{Acknowledgments}

The authors thank Dr. Tatsuro Irimura, Kaori DendaNagai, and Miki Noji for providing the cell lines used in this study. They thank Dr. Benjamin Cravatt (The Scripps Research Institute, La Jolla, CA) for providing the fluorophosphonate-rhodamine chemical probe. They thank the members of the Laboratory of Proteomics and Biomolecular Science, members of the Laboratory of Molecular and Biochemical Research, Research Support Center, Juntendo University Graduate School of Medicine, Tokyo, Japan, and Yumiko Sato for technical assistance. This work was supported by the Ministry of Education, Culture, Sports, Science, and Technology- (MEXT-) supported program for "Fostering Global Physicians - Reform of Medical Education by Balancing Basic Research and Clinical Practice" (M.I.) and by Grant-in-Aid for Scientific Research (KAKENHI) from the MEXT of the Japan Society for the Promotion of Science (JSPS) (Grant nos. $15 \mathrm{H} 06600$ to Hyeon-Cheol Lee, 15H05904, and 15H04708 to Takehiko Yokomizo). This study was supported in part by a Grantin-Aid (S1311011 and S1511008L) from the Foundation of Strategic Research Projects in Private Universities from the MEXT and a grant of the Institute for Environmental and Gender-Specific Medicine.

\section{Supplementary Materials}

Table S1: PCR primers used for TP53 sequencing. Table S2: colorectal cancer specimens analyzed in this study. Figure S1: p21 expression in human colorectal cancer and adjacent normal tissues was quantified by real-time reverse transcriptase PCR. (a) p21 mRNA was significantly decreased in the tumor tissue. 18S rRNA was used as a reference gene. ${ }^{* *} P<0.001$ by paired two-tailed $t$-test. (b) The p21 expression levels were compared between tumors with TP53 mutations generating nonfunctional p53 protein and tumors without TP53 mutations. ${ }^{* * *} P<0.001$. A TukeyKramer test was used. Table S3: correlation between p21 and CES2 expression. (Supplementary Materials)

\section{References}

[1] R. Humerickhouse, K. Lohrbach, L. Li, W. F. Bosron, and M. E. Dolan, "Characterization of CPT-11 hydrolysis by human liver carboxylesterase isoforms hCE-1 and hCE-2," Cancer Research, vol. 60, no. 5, pp. 1189-1192, 2000.

[2] S. P. Sanghani, S. K. Quinney, T. B. Fredenburg et al., "Carboxylesterases expressed in human colon tumor tissue and their role in CPT-11 hydrolysis," Clinical Cancer Research, vol. 9, no. 13, pp. 4983-4991, 2003.

[3] M. Xie, D. Yang, L. Liu, B. Xue, and B. Yan, "Human and rodent carboxylesterases: immunorelatedness, overlapping substrate specificity, differential sensitivity to serine enzyme inhibitors, and tumor-related expression," Drug Metabolism and Disposition, vol. 30, no. 5, pp. 541-547, 2002.

[4] G. Xu, W. Zhang, M. K. Ma, and H. L. McLeod, "Human carboxylesterase 2 is commonly expressed in tumor tissue and is correlated with activation of irinotecan," Clinical Cancer Research, vol. 8, no. 8, pp. 2605-2611, 2002.

[5] J. G. Slatter, L. J. Schaaf, J. P. Sams et al., "Pharmacokinetics, metabolism, and excretion of irinotecan (CPT-11) following I.V. Infusion of [(14)C]CPT-11 in cancer patients," Drug Metabolism and Disposition, vol. 28, no. 4, pp. 423-433, 2000.

[6] W. Choi, D. Cogdell, Y. Feng, S. R. Hamilton, and W. Zhang, "Transcriptional activation of the carboxylesterase 2 gene by the p53 pathway," Cancer Biology \& Therapy, vol. 5, no. 11, pp. 1450-1456, 2006.

[7] D. Xiao, D. Yang, L. Guo, W. Lu, M. Charpentier, and B. Yan, "Regulation of carboxylesterase-2 expression by p53 family proteins and enhanced anti-cancer activities among 5-fluorouracil, irinotecan and doxazolidine prodrug," British Journal of Pharmacology, vol. 168, no. 8, pp. 1989-1999, 2013.

[8] A. J. Munro, S. Lain, and D. P. Lane, "P53 abnormalities and outcomes in colorectal cancer: a systematic review," British Journal of Cancer, vol. 92, no. 3, pp. 434-444, 2005.

[9] S. I. Matsui, M. A. Arredondo, C. Wrzosek, and Y. M. Rustum, "DNA damage and p53 induction do not cause ZD1694induced cell cycle arrest in human colon carcinoma cells," Cancer Research, vol. 56, no. 20, pp. 4715-4723, 1996.

[10] Y. Liu and W. F. Bodmer, "Analysis of P53 mutations and their expression in 56 colorectal cancer cell lines," Proceedings of the National Academy of Sciences of the United States of America, vol. 103, no. 4, pp. 976-981, 2006.

[11] S. Djelloul, M.-E. Forgue-Lafitte, B. Hermelin et al., "Enterocyte differentiation is compatible with SV40 large T expression and loss of p53 function in human colonic Caco-2 cells," FEBS Letters, vol. 406, no. 3, pp. 234-242, 1997.

[12] T. Okamoto, H. Izumi, T. Imamura et al., "Direct interaction of $\mathrm{p} 53$ with the Y-box binding protein, YB-1: a mechanism for regulation of human gene expression," Oncogene, vol. 19, no. 54, pp. 6194-6202, 2000.

[13] P. J. Rochette, N. Bastien, J. Lavoie, S. L. Guérin, and R. Drouin, "SW480, a p53 double-mutant cell line retains proficiency for some p53 functions," Journal of Molecular Biology, vol. 352, no. 1, pp. 44-57, 2005.

[14] L. Bouaoun, D. Sonkin, M. Ardin et al., "TP53 variations in human cancers: new lessons from the IARC TP53 database and genomics data," Human Mutation, vol. 37, no. 9, pp. 865-876, 2016.

[15] L. T. Vassilev, B. T. Vu, B. Graves et al., "In vivo activation of the p53 pathway by small-molecule antagonists of MDM2," Science, vol. 303, no. 5659, pp. 844-848, 2004.

[16] J. K. Sax and W. S. El-Deiry, "p53 downstream targets and chemosensitivity," Cell Death \& Differentiation, vol. 10, no. 4, pp. 413-417, 2003.

[17] A. Willis, E. Jung, T. Wakefield, and X. Chen, "Mutant p53 exerts a dominant negative effect by preventing wild-type p53 from binding to the promoter of its target genes," Oncogene, vol. 23, no. 13, pp. 2330-2338, 2004.

[18] F. Vikhanskaya, M. K. Lee, M. Mazzoletti, M. Broggini, and K. Sabapathy, "Cancer-derived p53 mutants suppress p53target gene expression-potential mechanism for gain of function of mutant p53," Nucleic Acids Research, vol. 35, no. 6, pp. 2093-2104, 2007. 
[19] D. Menendez, A. Inga, and M. A. Resnick, "The expanding universe of p53 targets," Nature Reviews Cancer, vol. 9, no. 10 , pp. 724-737, 2009.

[20] G. M. Simon and B. F. Cravatt, "Activity-based proteomics of enzyme superfamilies: serine hydrolases as a case study," Journal of Biological Chemistry, vol. 285, no. 15, pp. 1105111055, 2010.

[21] A. L. Gartel and A. L. Tyner, "Transcriptional regulation of the p21(WAF1/CIP1) gene," Experimental Cell Research, vol. 246, no. 2, pp. 280-289, 1999.

[22] V. Bykov, N. Issaeva, A. Shilov et al., "Restoration of the tumor suppressor function to mutant p53 by a low-molecular-weight compound," Nature Medicine, vol. 8, no. 3, pp. 282-288, 2002.

[23] V. Bykov and K. G. Wiman, "Mutant p53 reactivation by small molecules makes its way to the clinic," FEBS Letters, vol. 588, no. 16, pp. 2622-2627, 2014.

[24] X. Yu, S. Narayanan, A. Vazquez, and D. R. Carpizo, "Small molecule compounds targeting the $\mathrm{p} 53$ pathway: are we finally making progress?"” Apoptosis, vol. 19, no. 7, pp. 1055-1068, 2014.

[25] X. L. Li, J. Zhou, Z. R. Chen, and W. J. Chng, "P53 mutations in colorectal cancer - molecular pathogenesis and pharmacological reactivation," World Journal of Gastroenterology, vol. 21, no. 1, pp. 84-93, 2015.

[26] S. Lehmann, V. Bykov, D. Ali et al., "Targeting p53 in vivo: a first-in-human study with p53-targeting compound APR-246 in refractory hematologic malignancies and prostate cancer," Journal of Clinical Oncology, vol. 30, no. 29, pp. 3633-3639, 2012.

[27] W. Shang, J. Liu, R. Chen et al., "Fluoxetine reduces CES1, CES2, and CYP3A4 expression through decreasing PXR and increasing DEC1 in HepG2 cells," Xenobiotica, vol. 46, no. 5, pp. 393-405, 2015.

[28] S. E. Pratt, S. Durland-Busbice, R. L. Shepard, K. HeinzTaheny, P. W. Iversen, and A. H. Dantzig, "Human carboxylesterase-2 hydrolyzes the prodrug of gemcitabine (LY2334737) and confers prodrug sensitivity to cancer cells," Clinical Cancer Research, vol. 19, no. 5, pp. 1159-1168, 2013.

[29] S. K. Quinney, S. P. Sanghani, W. I. Davis et al., "Hydrolysis of capecitabine to $5^{\prime}$-deoxy-5-fluorocytidine by human carboxylesterases and inhibition by loperamide," Journal of Pharmacology and Experimental Therapeutics, vol. 313, no. 3, pp. 1011-1016, 2005.

[30] B. L. Barthel, Z. Zhang, D. L. Rudnicki et al., "Preclinical efficacy of a carboxylesterase 2-activated prodrug of doxazolidine," Journal of Medicinal Chemistry, vol. 52, no. 23, pp. 7678-7688, 2009. 


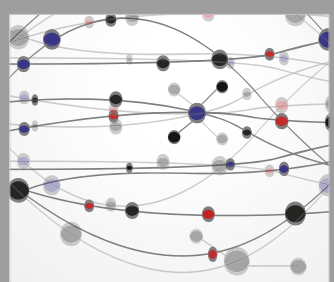

The Scientific World Journal
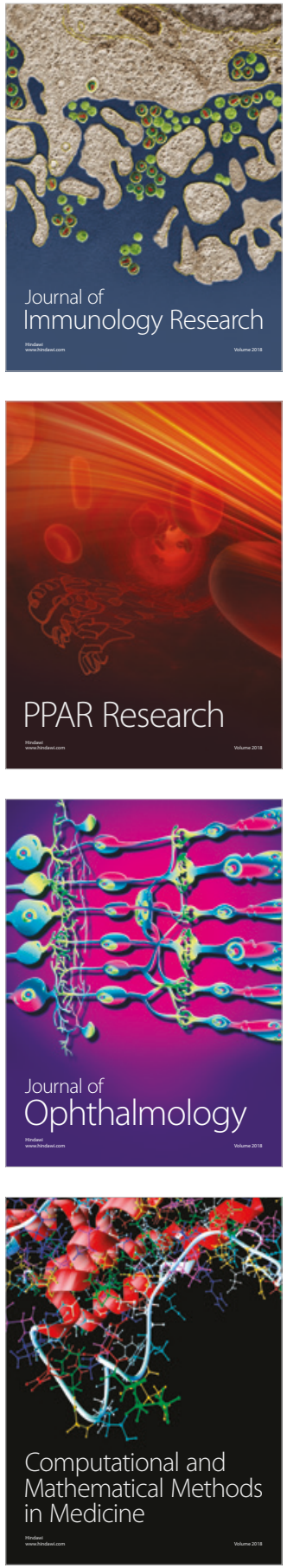

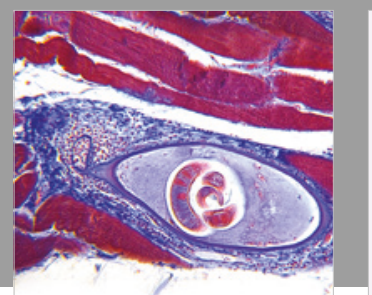

Gastroenterology Research and Practice

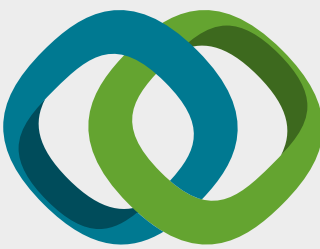

\section{Hindawi}

Submit your manuscripts at

www.hindawi.com
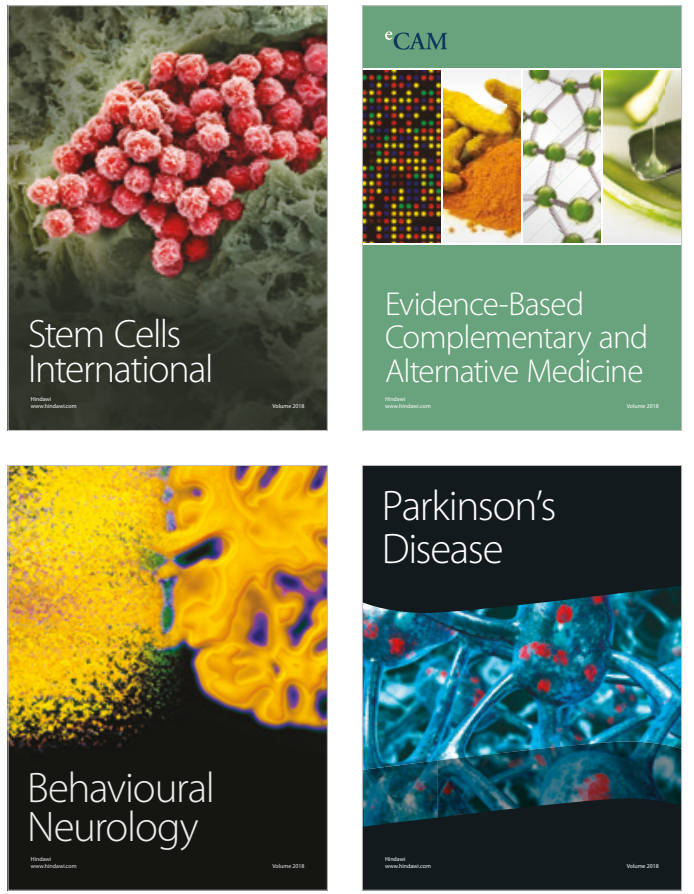

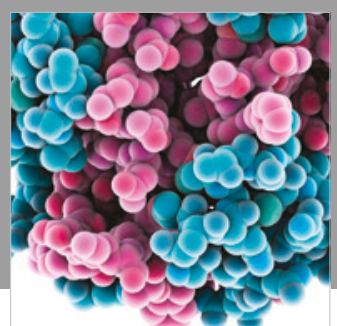

ournal of

Diabetes Research

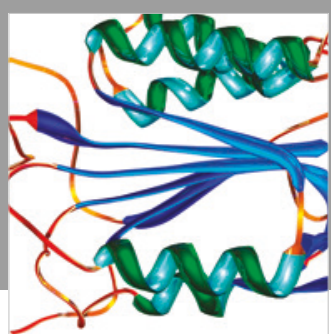

Disease Markers
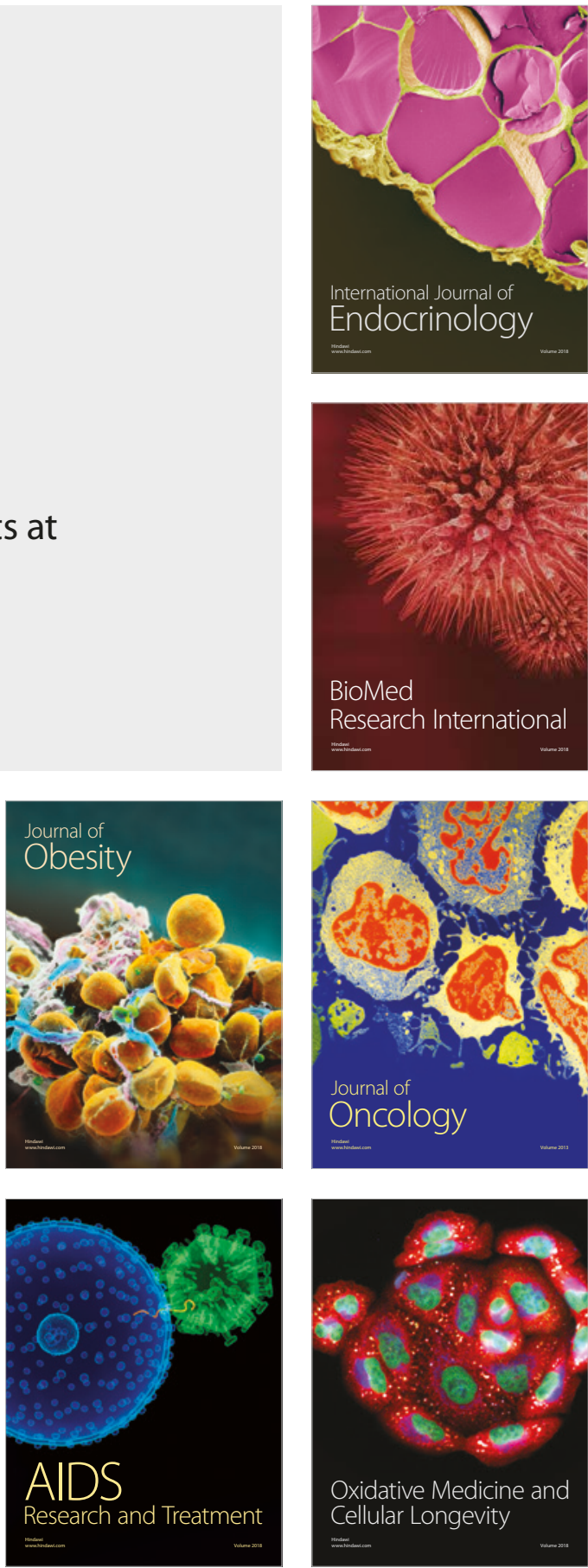